Review

\title{
Pathology in Continuous Infusion Studies in Rodents and Non-Rodents and ITO (Infusion Technology Organisation)- Recommended Protocol for Tissue Sampling and Terminology for Procedure-Related Lesions
}

\author{
Klaus Weber ${ }^{1}$, Vasanthi Mowat ${ }^{2}$, Elke Hartmann³, Tanja Razinger ${ }^{4}$, Hans-Jörg Chevalier ${ }^{1}$, \\ Kai Blumbach ${ }^{4}$, Owen P. Green 5 , Stefan Kaiser ${ }^{4}$, Stephen Corney ${ }^{4}$, Ailsa Jackson ${ }^{4}$, and \\ Agustin Casadesus ${ }^{6}$ \\ ${ }^{1}$ AnaPath GmbH, Buchsweg 56, 4625 Oberbuchsiten, Switzerland \\ ${ }^{2}$ Huntingdon Life Sciences, Huntingdon, Cambs, UK \\ ${ }^{3}$ Bayer Schering Pharma AG, BSP-GDD-GED-TOX-P\&CP, 42096 Wuppertal, Germany \\ ${ }^{4}$ Harlan Laboratories Ltd., Zelgliweg 1, 4452 Itingen, Switzerland \\ ${ }^{5}$ LeVerts Ltd Consultancy, Peterborough, UK \\ ${ }^{6}$ Harlan Laboratories S.A., Centro Industrial Santiga, c/Argenters, 6, 08130 Santa Perpetua de Mogoda (Barcelona), España
}

\begin{abstract}
Many variables may affect the outcome of continuous infusion studies. The results largely depend on the experience of the laboratory performing these studies, the technical equipment used, the choice of blood vessels and hence the surgical technique as well the quality of pathological evaluation. The latter is of major interest due to the fact that the pathologist is not involved until necropsy in most cases, i.e. not dealing with the complicated surgical or in-life procedures of this study type. The technique of tissue sampling during necropsy and the histology processing procedures may influence the tissues presented for evaluation, hence the pathologist may be a source of misinterpretation. Therefore, ITO proposes a tissue sampling procedure and a standard nomenclature for pathological lesions for all sites and tissues in contact with the port-access and/or catheter system. (DOI: 10.1293/tox.24.113; J Toxicol Pathol 2011; 24: 113-124)
\end{abstract}

Key words: continuous infusion, pathology, beagle, non-rodents, rodents, tissue sampling, terminology

\section{Introduction}

There are a number of relevant factors to be considered when entering a preclinical development program with a compound intended for intravenous infusion in the clinics. Those factors include the choice of species, the related choice of the infusion technique and hence, the surgical method. In addition, there are many potential influences such as chemical composition of the catheter material, the type of catheterized blood vessel and its calibre, as well as the maintenance of catheter patency during the in-life period. At necropsy, the techniques of tissues sampling, preservation and histology are important factors to consider during differentiation of method-related, postsurgical and

(C2011 The Japanese Society of Toxicologic Pathology

Received: 19 March 2011, Accepted: 21 March 2011

Mailing address: Klaus Weber, Harlan Laboratories Ltd., Zelgliweg

1, 4452 Itingen, Switzerland

TEL: 41-0-61 9751268

E-mail:kweber@harlan.com traumatic local alterations or even infectious conditions from test item-induced lesions. Further complications may occur due to lesions that are simply enhancements of the background of pre-existing focal alterations or background lesions in other organs. In such cases it could be difficult, and in some cases even impossible, to distinguish between a range of pre-existing conditions and lesions attributable to treatment.

The longer the in-life part of a study the more problems that may occur. All negative circumstances during surgery and the in-life period may enhance pre-existing lesions. Test item incompatibility to the catheter material may cause further complications. The catheter material by itself may cause damage to invaded blood vessels, and, in addition, inflammatory or degenerative lesions may be enhanced by adverse test item related effects. Therefore, an inexperienced pathologist may be also an important source of misinterpretation of study results.

A well performed continuous infusion study should not result in pain or loss of animals, even as the worst consequences of poor practice. The workload should stay within 


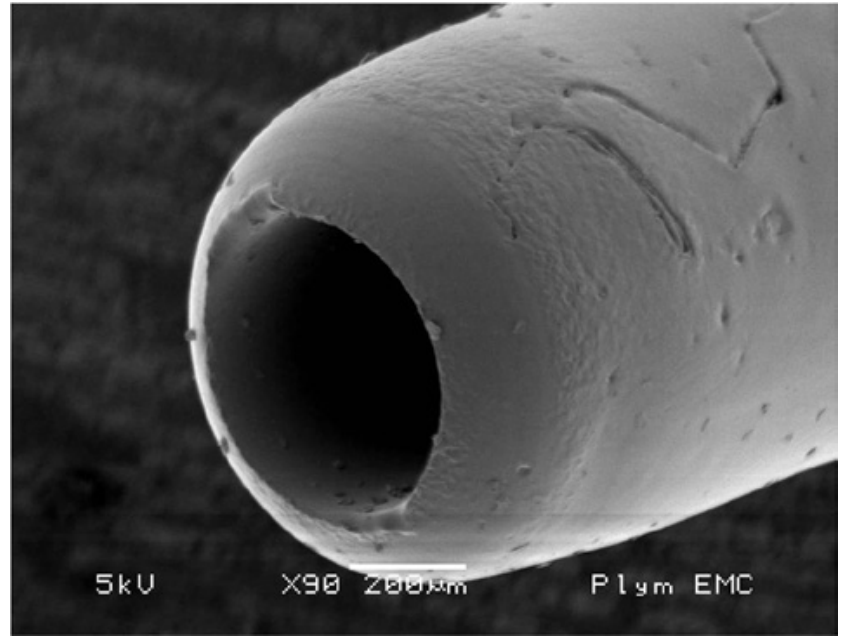

Fig. 1. Round tip catheter (scanning electron microscopy).

an expected range. The normal, expected background pathology should not be enhanced by avoidable method-related complications and, at the end of a study, the differentiation between traumatic and post-surgical lesions vs test item induced lesions must be possible.

The Infusion Technology Organization (ITO) was founded in 2009 and registered in Switzerland by representatives from CROs and the pharmaceutical industry (www. ito-org.com) to facilitate the exchange of knowledge on infusion techniques in the pre-clinical and clinical field. At the first annual international meeting in Manchester in 2009, the necessity for a uniform tissue sampling procedure for method-related tissues and for a harmonized nomenclature was considered to be an important step to reach a common understanding of lesions related to the preclinical continuous infusion studies. The present article is a shared opinion of pathologists and toxicologists being members of ITO and was original published in the internal journal of ITO 'The Infusionist', as proceedings of the first international meeting in Manchester, 2010.

\section{Factors Influencing Method-Related Pathology: Choice of Infusion Technique, Species and Re- lated Issues}

Generally, the choice of the infusion technique includes free transdermal catheters in blood vessels or securing subcutaneous buttons with or without usage of a harness or jackets vs tail cuff techniques in small animals. On the other hand port access systems vs transdermal catheters are used today in larger animals. Depending on the technique applied the range of induced lesions might vary slightly from study to study.

When choosing the relevant species for the preclinical development of a product for continuous infusion, it should be considered, that any species has advantages and disadvantages when used for this route of administration.
For example, rats are small but fast growing animals with a low arousal threshold. Mice may be considered as the most complicated surgical model, but successful studies of up to 28 days of treatment are reported ${ }^{8}$. Rabbits are larger and therefore an easier model with respect to surgery; however, they are very nervous and anxious animals. In rabbits, the corpuscular hematocrit is also very different from other species, and there are different blood cell populations, e.g. heterophils. Furthermore, the rabbit jugular vein is very short, and hence the distance to the heart orifices is often underestimated. In contrast dogs and monkeys are large animals, providing an easy access to blood vessels. However, there are also disadvantages in these animal models which are the introduced traumatic, long subcutaneous catheter tunnels, the subcutaneous port systems covered by a shirt (monkeys) and/or jacket above the skin, and the long hair that causes skin irritation due to trauma induced by the jackets. Moreover, dogs and monkeys should not be expected to be quiet patients after surgery. In contrast, they recover very shortly after surgery, i.e. they are very active, playing, scratching and biting. The latter makes it almost impossible to use peripheral veins of the legs as indwelling points for catheters.

\section{Factors Influencing Method-Related Pathology: Choice of Port and Catheter Material and Selec- tion of Blood Vessel Type}

It is important to check the compatibility of the test item to the port system and catheter material prior to performing a study. Adhesion of the test item to the catheter material or sludge formation within the port cavity due to precipitation or any other phenomenon induced due to unexpected interactions between test item and involved materials (e.g. also the vehicle) might cause premature termination of the study. This can become necessary due to a fully blocked catheter-port system, the formation of emboli or anaphylactic reactions due to sludge formation flushed into the blood stream.

The catheters are usually made of silicone, polyurethane (PU) or polyethylene (PE). It should be pointed out that in several studies performed by different authors the PE-catheters were found to completely lose their flexibility and become brittle within less than 2 weeks after implantation. Therefore a high risk of an increased incidence of local vascular injuries and flushing of released particles into the lung is likely when using PE catheters. Many scientists have had this experience with PE and therefore the majority of infusion studies these days are performed using PU-catheters and, to a lower extent, also silicone catheters.

Only sterile catheter materials should be used. 'Off the roll'-material cannot be considered fully sterile without difficult sterilization processes and also, tips of selfcut catheters are sharp-edged and induce severe lesions as early as during surgery. Hence these "self-made" catheters should not be used. Catheters should have a round tip (Fig. 1). Several investigations have been performed on this issue. Fredenburg ${ }^{1}$ demonstrated a highly significant increase 


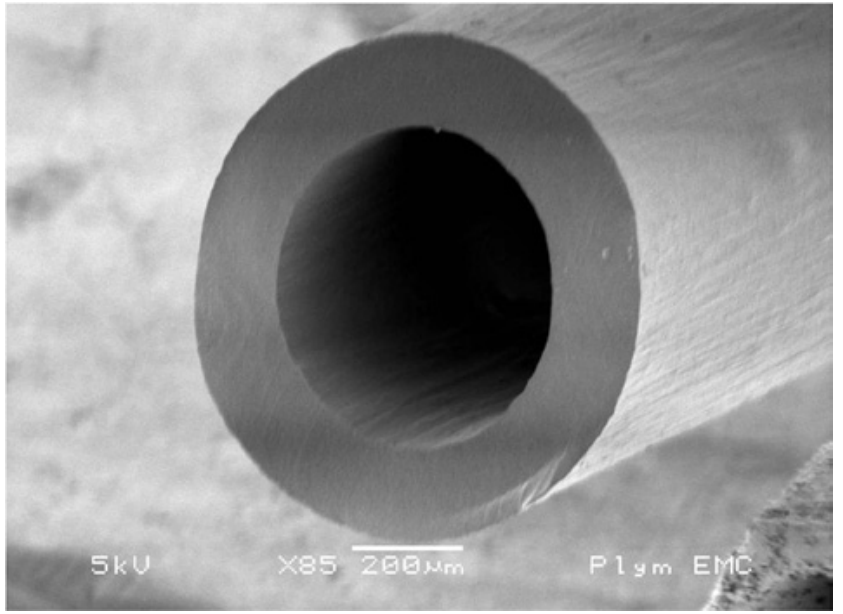

Fig. 2. Blunt tip catheter (scanning electron microscopy).

in patency time for catheters with rounded tips (mean 27.8 days) vs blunt tip catheters (Fig. 2) (mean 19.9 days) without rinsing, whereas a lock solution was not of significant influence on patency.

Catheter patency has to be maintained during the course of the study. This may be achieved by daily continuous rinsing with the vehicle. Sludge formation in port systems should be avoided by slightly pulsatile flushing or removed by aspiration before the next intermittent administration. Catheter rinsing with the vehicle containing anticoagulants such as heparin may affect the study outcome and hence should be avoided. By influencing the test system the resulting data generated might lead to wrong conclusions for the test item's toxicological profile. When using port systems with lock solutions, the lock solution should not be pushed into the system. Experience shows that rinsing with saline should be done frequently during the pre-test period. Especially in dogs, it may be considered that endothelium has a very high activity. By its intrinsic behavior and ability to cover the surface of blood vessels (geometrically hollow tubes), the endothelial cells grow into the catheter lumen, predisposing to small thrombi that may finally lead to a loss of patency.

In a validation study performed at Harlan Laboratories Ltd., Switzerland, round soft tip polyurethane catheters secured by an Instech Solomon Vascular Access Harnesses (VAH) were found to be the best combination in studies with treatment duration of 13 weeks. The PU-catheter was much better tolerated than silicon catheters and the harness used was superior to the normal Infusion Harness (IH). The latter was mainly due to the fact, that a disconnection of the infusion line, frequently seen at the connector when using the $\mathrm{IH}$, is not possible with the septum functionality of the VAH. Repeated re-connection and related handling of the implanted catheter (up to $4 \mathrm{x}$ in an 28-day study) increased the incidence and severity of inflammatory processes in catheterized blood vessels resulting in a high number of animals prematurely leaving the study ${ }^{4}$.

The port system itself may be a problem when port bodies are made from plastic instead of stainless steel. When using ports made of Teflon, foreign bodies are occasionally seen in the blood vessel wall near the point of compound delivery; these are most likely scratched out by the cannula at the port bottom during assembly. Other foreign bodies of unknown nature and origin are occasionally visible along the course of the indwelling catheter and are considered to represent catheter or septum material. Such foreign bodies with inflammatory reactions may be another source of unwanted background pathology and have been described by others too ${ }^{3}$.

The selection of the blood vessel is a very important factor. Different parameters such as its calibre are more important in smaller animals, but minimizing manipulation of the external part of the catheter by the animal in larger animals is an important factor which contributes to a successful study. It is for example almost impossible to get a study performed in dogs for more than 3 days using the saphenous vein because the animals scratch even the best covered wounds and quickly pull the catheter out of the blood vessels.

The relation between vascular and catheter diameter is a source of method-related pathology and causes different lesions in small vs large animals. In small animal models, the vascular diameter may even cause the exclusion of single animals or a whole strain (in mice) of a study. In rabbits, it must be considered that the length of the jugular vein is often overestimated and hence, catheters are misplaced into the heart or even lungs.

In pharmacological or pharmacodynamic studies, it may be important to get access to arteries. From our experience, in short term studies where catheters in dogs were placed into peripheral arteries, the normal movement of the animals combined with the blood pressure were recognized as factors which pushed out the catheters from arteries.

\section{Surgery and Related Issues}

In any study with any species, the professional skills of the surgeon can be judged at the end of a study by histopathological evaluation. Besides individual practical skills, there are circumstances affecting the study outcome, such as unnecessary, locally harmful vascular damage by sharp tipped catheters (e.g. self-made catheters mentioned above), excessive ligatures for the catheter fixation, or even the use of glue to fix a catheter in the blood vessel. There are many techniques that can be used to avoid such harmful events, e.g. the use of elastic bands when handling blood vessels during surgery to avoid further tissue damage or excessive blood loss.

In small animal models, it should be considered that usually there is only one chance to introduce a catheter into the small calibre blood vessel. However, even in larger animals like dogs, the repeated introduction of catheters into the blood vessel should be avoided whenever possible to 


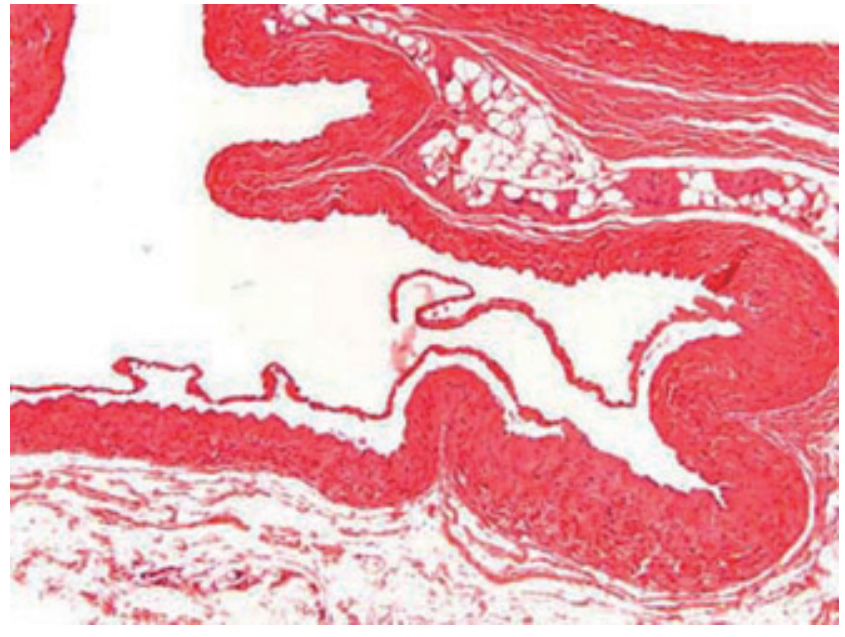

Fig. 3. Normal control jugular vein $(\operatorname{dog}, \mathrm{HE}$, lens $\times 10)$.

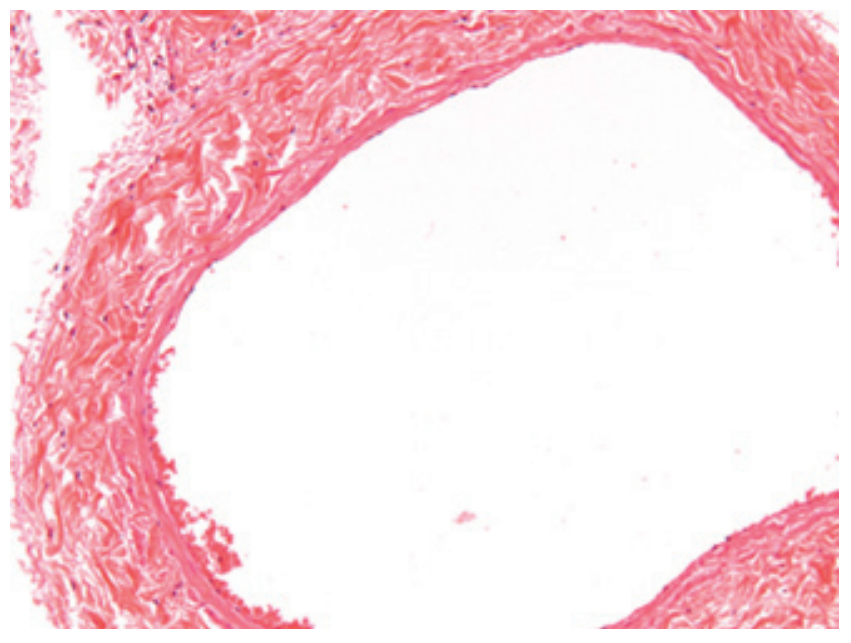

Fig. 4. Normal vena cava (rat, HE, lens $\times 20$ ).

avoid unnecessary sloughing of endothelial cells.

In larger animals like dogs, under certain circumstances (loss or pulling out of catheter, broken catheters, necrosis, thrombosis etc.) infusion sites may be changed during the course of a study; as has been done in studies performed at Harlan Laboratories Ltd., Switzerland. This is not considered to invalidate a study. However, the related surgical procedures and pharmacological impact need to be documented and explained in the context of the general treatment during the study.

\section{Tissue sampling}

It is highly recommended to sample tissues at the following locations in large and small animals:

- dermal subcutaneous tissue around the implanted port or the site of catheter perforation of the skin in studies where no port access system was used,

- at least one sample of tissue surrounding the subcuta-

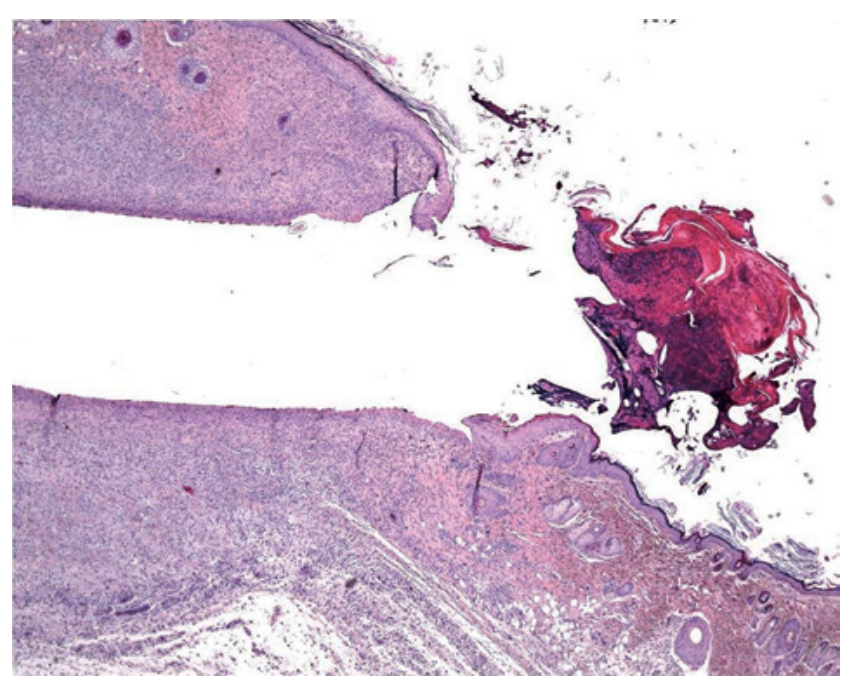

Fig. 5. Subcutaneous exteriorization of catheter (rat, HE, lens $\times 4$ ).

neous tunnel at the course of catheter,

- vascular and surrounding tissues at the point where the catheter indwells into the blood vessel,

- vascular and surrounding tissues at the tip of catheter, and

- two samples for:

rodents and rabbits at a distance of approximately: 0.3 and 0.5 to $1.0 \mathrm{~cm}$ distally to the point of delivery. non-rodents: 0.5 and $1.0 \mathrm{~cm}$ distally to the point of delivery

- control vessel (Figs. 3, 4).

The lesions that may be recorded at each of these sites are fairly consistent; varying within a small range only under well-established study conditions.

\section{Post-surgical and traumatic background lesions}

What is the normal background pathology in continuous infusion studies in dogs? A number of findings are consequences of surgery due to cutting into the tissue, haemorrhage, handling of blood vessels, fixing the catheters etc. Hence, tissue reaction and organization as remainders of organizing inflammation ranging from subacute to chronic or chronic active processes or scarring fibrosis with or without related hemorrhage and/or deposition of hemosiderin at all sites that were handled surgically need to be considered as normal reactive changes. This mainly affects the tissues surrounding the port or the transcutaneous catheter tunnel (Figs. 5, 6), the traumatically induced subcutaneous catheter tunnel, as well as the indwelling point of the catheter into the blood vessel and sites of ligatures. The information about the reactive and inflammatory changes at this site may have a great impact on the interpretation of lesions recorded at the regions around the tip of catheter and distal from it, e.g. extensively extending inflammation throughout the course of the catheter trail or systemic effects due to widespread infectious conditions. 


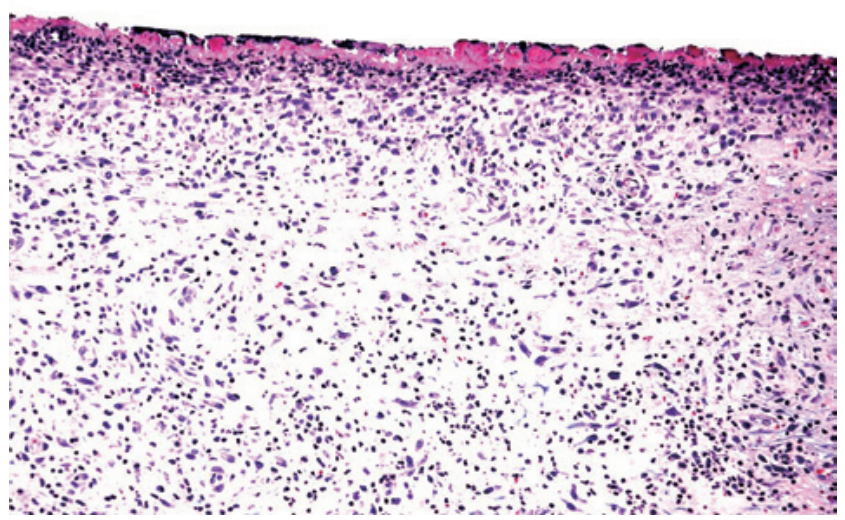

Fig. 6. Magnification of Fig. 5. Little inflammation in surrounding tissues (rat, HE, lens $\times 100$ ).

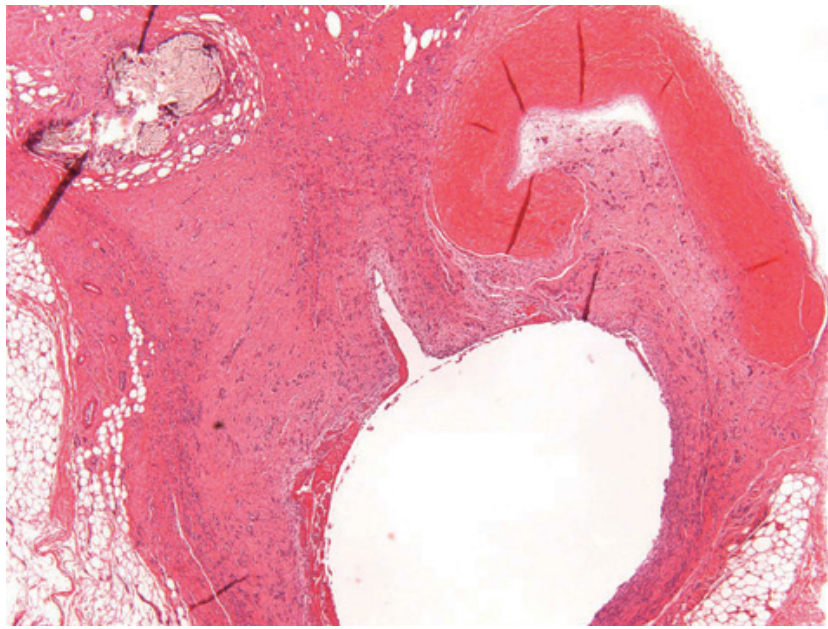

Fig. 7. Point of catheter indwelling into the jugular vein. Fibrotic scar covers cut. Cut off ends of vein rolls into lumen. Slight inflammation as indicator of scaring is a healing process (dog, HE, lens $\times 4$ )

At the indwelling point of the catheter into the blood vessel, there is always a reactive change caused by the organization of the tissue at the insertion point. This lesion consists of perivascular and vascular chronic inflammation and/or fibrosis along with scar tissue formation. At the site where the blood vessel was cut, the vessel walls turn to contract and the remnant stumps winds into the vessel lumen (Fig. 7). The indwelling catheter that now fills this cut-related vascular cleft completely is surrounded within approximately 7 days by a young connective tissue. Suture material causes stitch granuloma formation wherever ligatures are used to fix the catheter in a blood vessel (Fig. 8). The severity of the inflammatory process is related to the

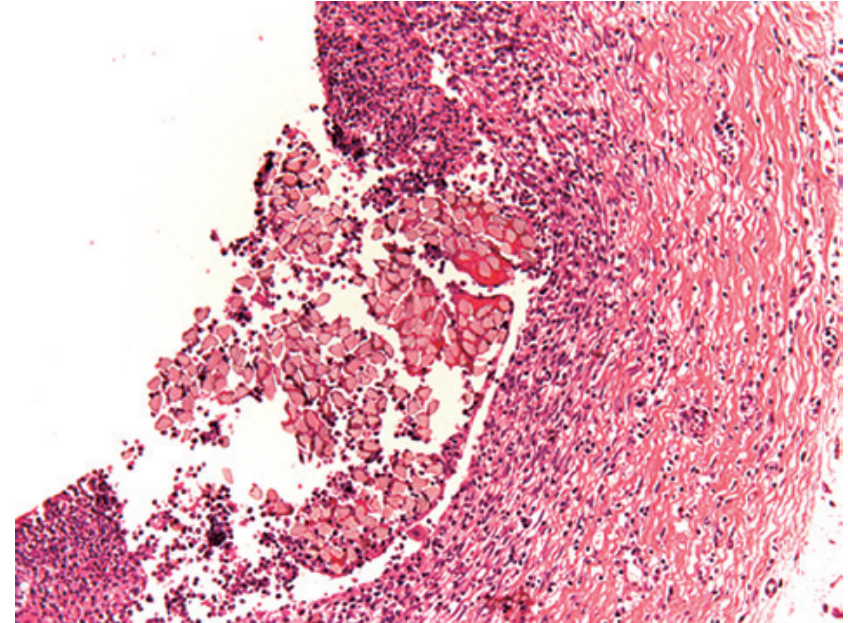

Fig. 8. Stitch granuloma in jugular vein. Note polyfile suture material appears at single threats surrounded by inflammatory cells (rat, HE, lens $\times 20$ ).

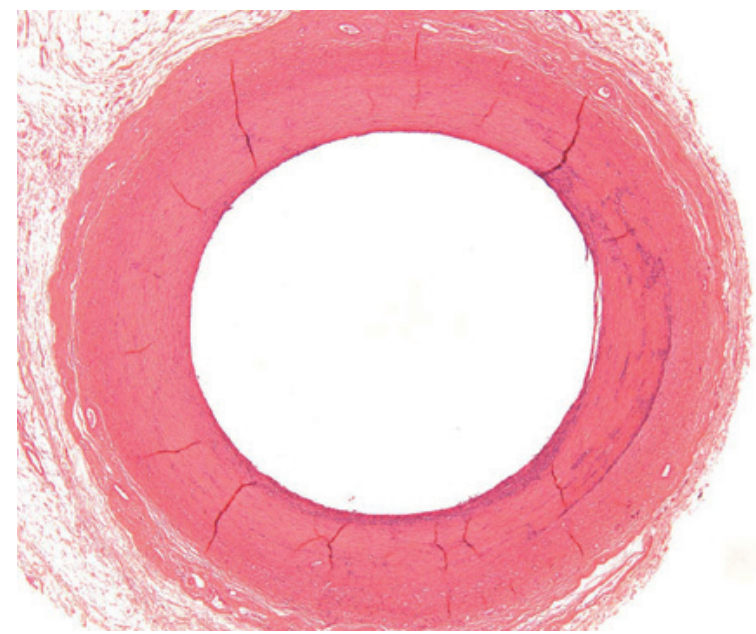

Fig. 9. Degenerated and fibrotic jugular vein wall at middle position of catheter tunnel. Note, that there is no periphlebitis and no active inflammatory process (dog, $\mathrm{HE}$, lens $\times 4$ ).

material used. Monofilament threads cause less inflammatory reactions than polyfilament materials. Monofilaments are surrounded by inflammatory cells consisting mainly of lymphocytes and macrophages (granulocytes are rare) and within approximately 7 days a thin rim of connective tissue surrounds the threads. A marked granulomatous reaction is a rare and unwanted event, but may be recorded 5-6 weeks after surgery 9 .

Along the course of the catheter within the blood vessel, a slight to marked inflammation restricted to the vessel wall and occasionally a slight to moderate perivascular inflammation may be recorded as expected background findings. Depending on the vascular and catheter diameters, a 


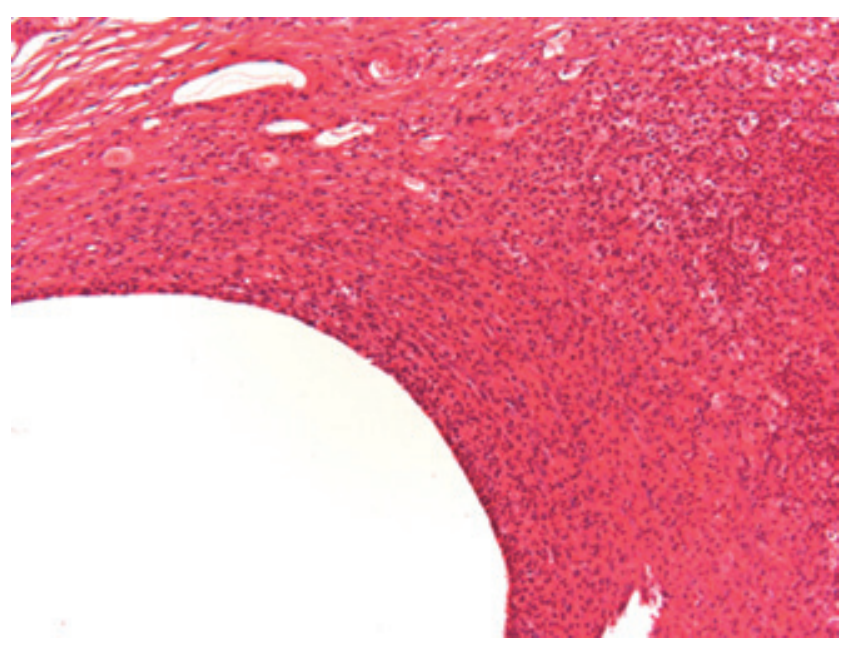

Fig. 10. Chronic inflammation and fibrosis of former jugular vein wall at middle position of catheter tunnel. Note, that there is no active inflammatory process (rat, $\mathrm{HE}$, lens $\times 20$ ).

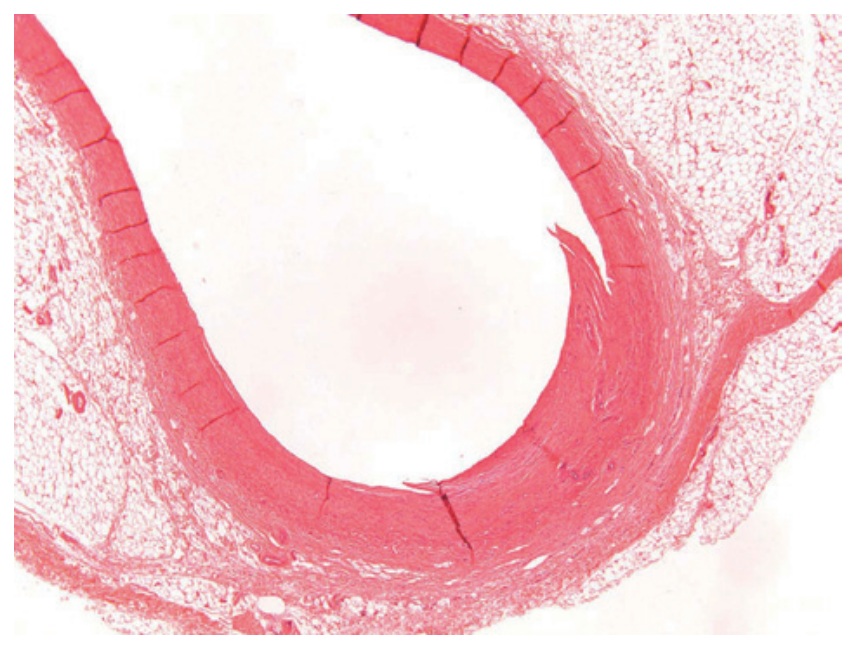

Fig. 11. Jugular vein at tip of catheter. Focal fibrotic and inflamed plug whereas the remaining part of vein is normal (dog, HE, lens $\times 4$ )

catheter in close contact with the blood vessel wall causes a fibrosing degeneration of the vessel wall (Figs. 9, 10). This however is not often encountered in dogs due to the large diameter of vessels used (e.g. jugular vein). In most cases the catheter is covered on its external surface by a sheath of fibrinous tissue or fibrin tags. Such sheaths may cover the external catheter surface completely from the catheter's indwelling point over the whole distance up to the tip of the catheter.

At the tip of catheter there is, in most cases, a minimal to slight (occasionally moderate) focal vasculitis and/or perivasculitis (Figs. 11, 12). Such inflammatory processes may be a very focal event, i.e. will be only recorded in a distinct segment of the blood vessel. This occurs most likely due to the placement of the catheter's tip, i.e. the tip of the cath-

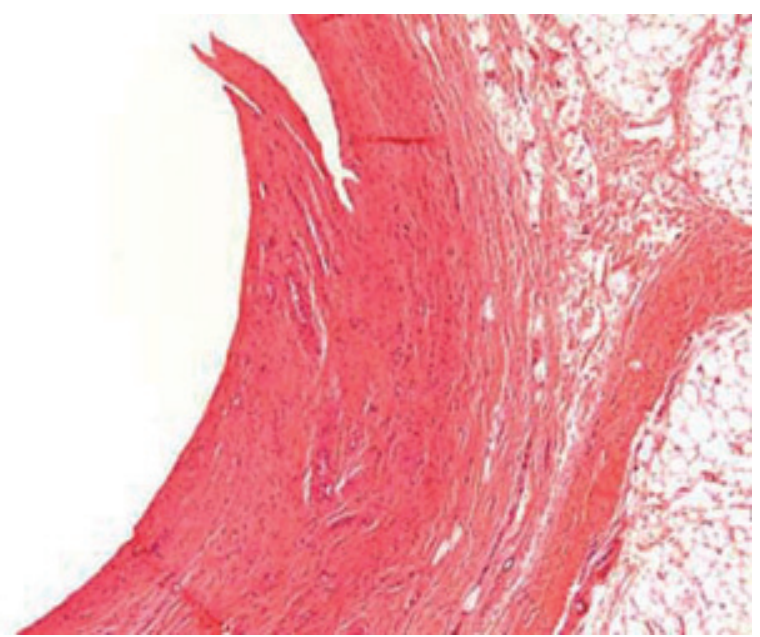

Fig. 12. Higher magnification of focal process as in Fig. 7 (dog, HE, lens $\times 10$ ).

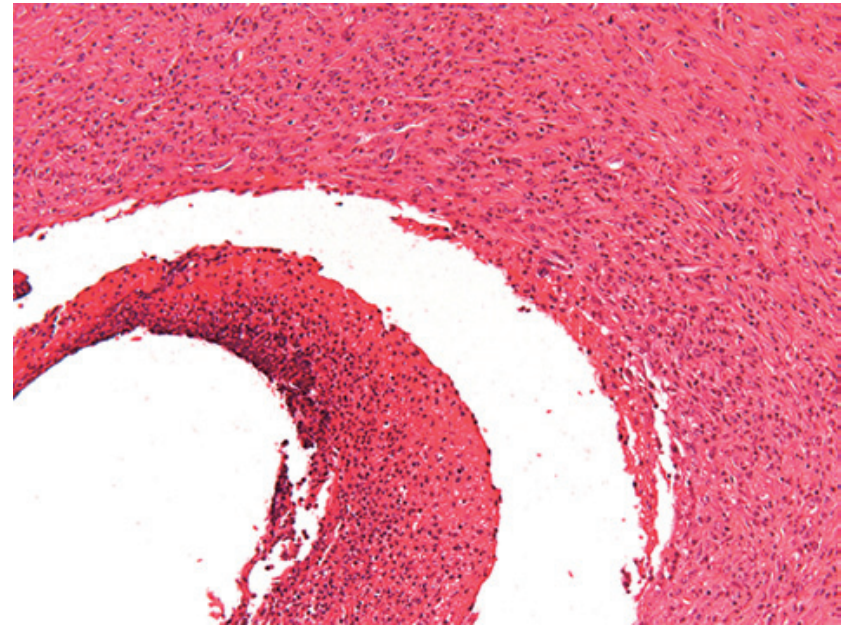

Fig. 13. Jugular vein at tip of catheter. Fibrotic vein wall and chronic inflammation as well as intraluminal pericatheteral fibrotic plug (rat, HE, lens $\times 20$ ).

eter moves slightly within the blood vessel and, like a whip, traumatizes the luminal surface. This trauma may produce a simple reactive change of the intima causing intimal proliferation (Fig. 17), characterized by a focal proliferation of endothelial cells along with the appearance of larger, round nuclei and a thickened submucosa. Depending on the type of blood vessel used, there may be a simple phlebitis or arteritis. This may lead to an endovasculitis characterized by an inflammatory infiltration of the intima, changes of the architecture and distortion of the normal morphology of the vascular intima by tissue proliferation along with a thickening of the submucosa and/or fibrosis (endophlebitis, endarteritis). In most cases however, the vessel wall is at least involved with the intima and media (phlebitis, arteritis). When the externa or adventitia becomes affected, there is 


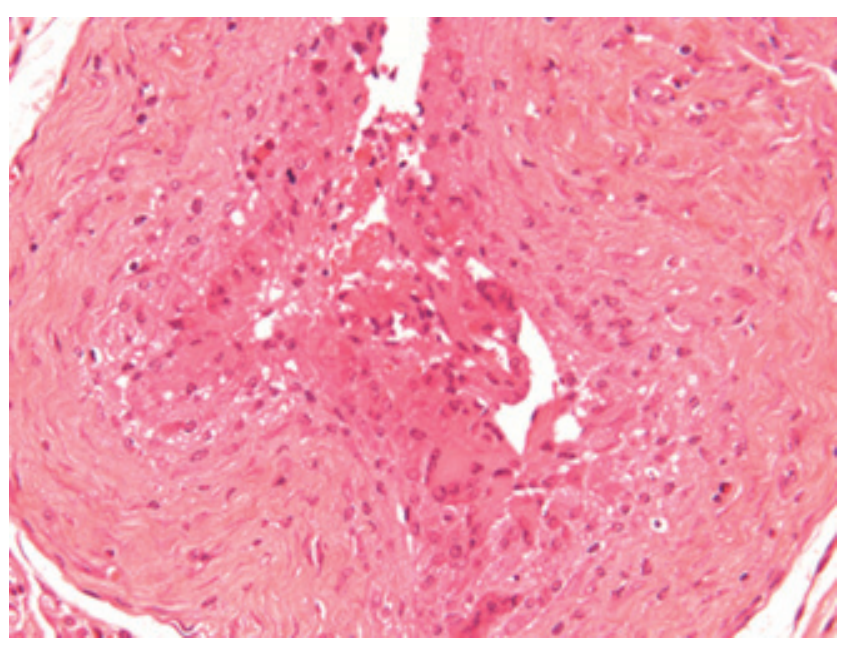

Fig. 14. Femoral vein near the tip of catheter. Thickened vein wall with intravascular inflammation (rat, HE, lens $\times 40$ ).

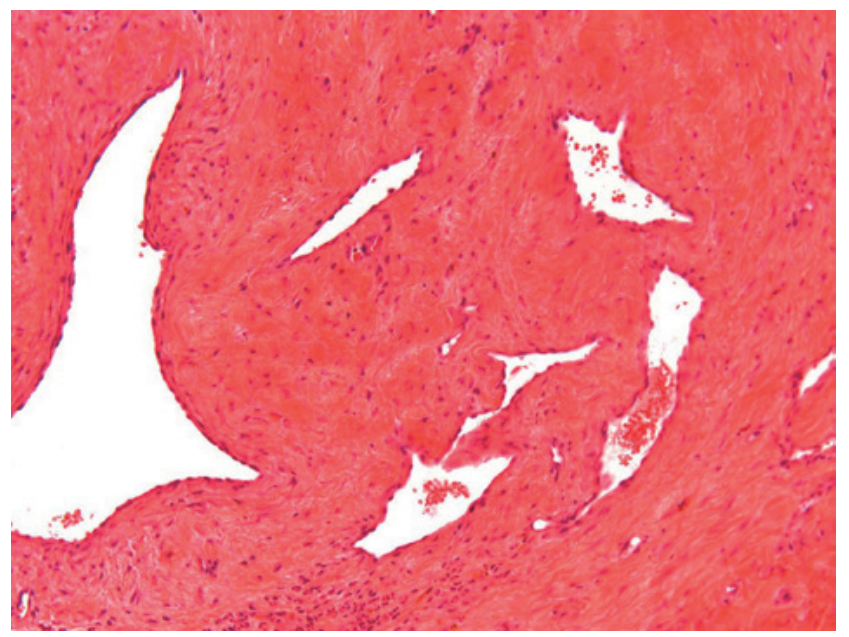

Fig. 15. Occluded and re-canalized jugular vein (thrombophlebitis) (dog, HE, lens $\times 20)$.

usually an accompanying perivasculitis extending into the surrounding connective tissues (periphlebitis, periarteritis) (Figs. 13, 14).

More often than a simple vasculitis, the occurrence of thrombi complicates the picture at this location (Fig. 15). Only rarely, a thrombus may be seen within the vascular lumen or superficially attached to it with no involvement of the vascular wall, and hence may be recorded as thrombus (Fig. 16). When along with vasculitis it is deemed to be of greater consistency to term the lesion thrombophlebitis or thrombarteritis. Thrombi may be fully occluding the vessel or not, and occasionally also may be recanalized.

A specific condition may be the isolated inflammation of a vein valve (Fig. 19). This is deemed to be a consequence of focal irritation of the vein valve due to contact with the

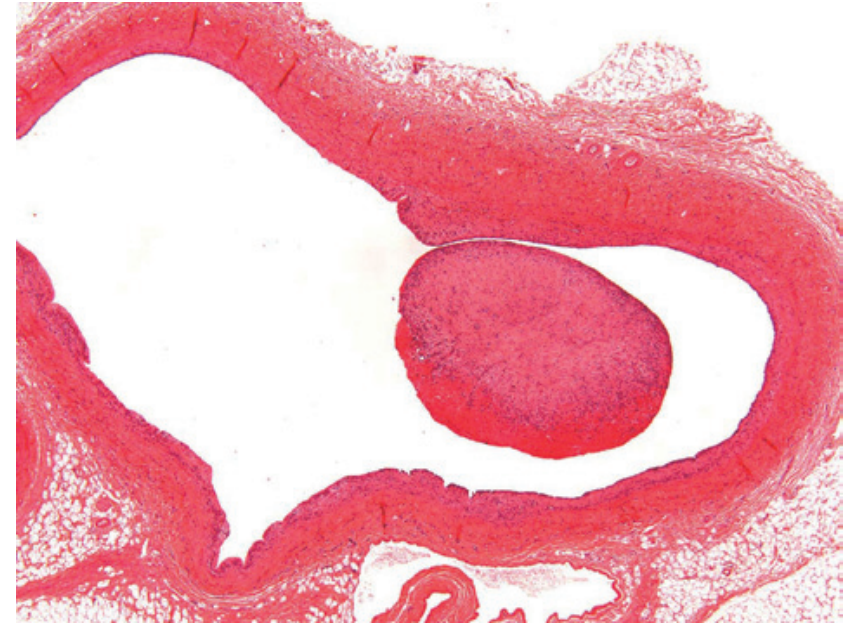

Fig. 16. Jugular vein with focal minimal inflammation and intraluminal thrombus near the tip of catheter. Thickened vein wall with intravascular inflammation (dog, HE, lens $\times 4)$.

catheter tip. It should be considered a focal endophlebitis (Fig. 18). Vascular or endovascular necrosis is not often encountered in large animal models at the location of the tip of catheter or distally from this point.

The blood vessel at a distance of 0.5 to $1 \mathrm{~cm}$ distal to the tip of catheter should be less affected than at the tip of catheter. In a number of cases, vasculitis/perivasculitis may still be observed in this region, but clearly at degrees of minor severity (Fig. 20). Inflammatory processes at higher severities at such sites need to be carefully excluded from being induced by the test item. They may be encountered occasionally in controls. In such cases, they are mainly caused by thrombosis/thrombovasculitis.

\section{Lesions Secondary to Post-Surgical and Trau- matic Conditions}

Secondary lesions related to postsurgical/-traumatic conditions may affect other organs or the complete organism. This is mainly due to the formation of focal thrombosis and secondary embolism. A small number of the animals in a study is always affected by such conditions and therefore has to be considered as normal within a defined incidence and severity range. The incidence and/or severity of lesions may increase in a procedure-related manner due to surgical methods and/or postsurgical care measurements but may be also enhanced by the test item properties. Most often, thrombi with related inflammatory lesions are recorded in lung and heart, and are less commonly seen in kidneys followed by other organs. In the heart, there may be endocardiosis in cases of increased thrombus formation (most likely by liberation of serotonin), endocarditis (especially valvular inflammation) (Figs. 21, 22), or myocardial inflammation (Fig. 23) induced by thrombi attached to the endocardium (Fig. 24). In extreme cases, even sepsis may occur related to 


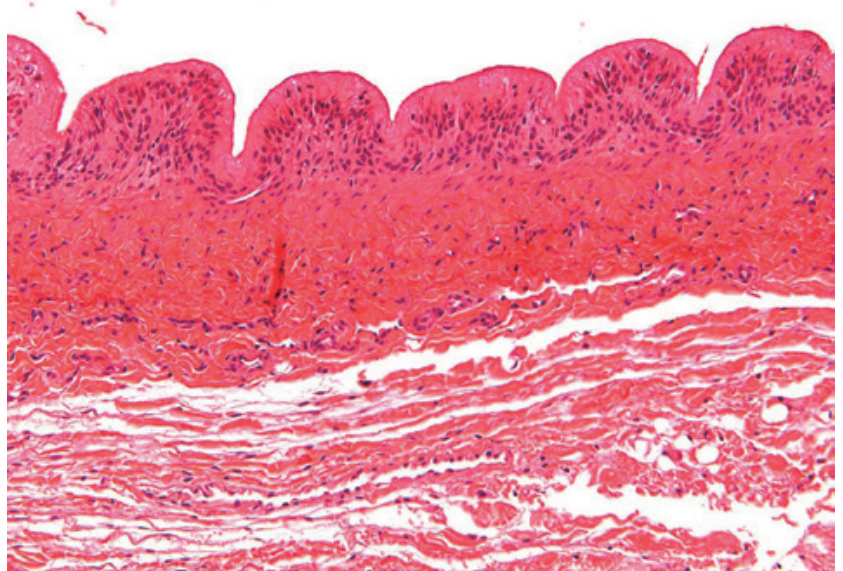

Fig. 17. Jugular vein with intimal proliferation around the tip of catheter $(\operatorname{dog}, \mathrm{HE}$, lens $\times 20)$.

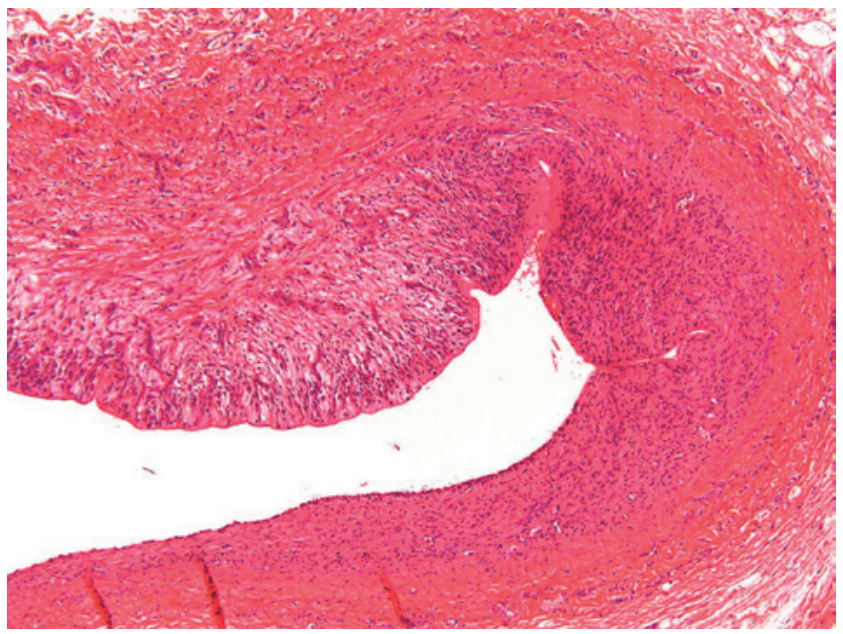

Fig. 18. Jugular vein at site of test item delivery with chronic endophlebitis (dog, HE, lens $\times 10)$.

both surgical procedures and post-surgical trauma or related to the test item, e.g. under treatment with immunosuppressive products. Extreme issues, such as unexpected precipitation of the test item in the catheter lumen, may be avoided by prior checking of their compatibility. There may be several reactive or stress-related lesions that are not of significant pathological value in rats, i.e. extramedullary erythropoiesis in the spleen, increased granulopoiesis in the bone marrow, adrenal cortical hypertrophy, thymus atrophy etc.

\section{Induced lesions}

Most of the induced lesions in blood vessels do not differ from previously described postsurgical/-traumatic lesions, except for their incidence and severity. The character of induced inflammation may be more aggressive, i.e. more severe and/or necrotizing. In some studies, test item may precipitate within the blood vessel and may form crystals in

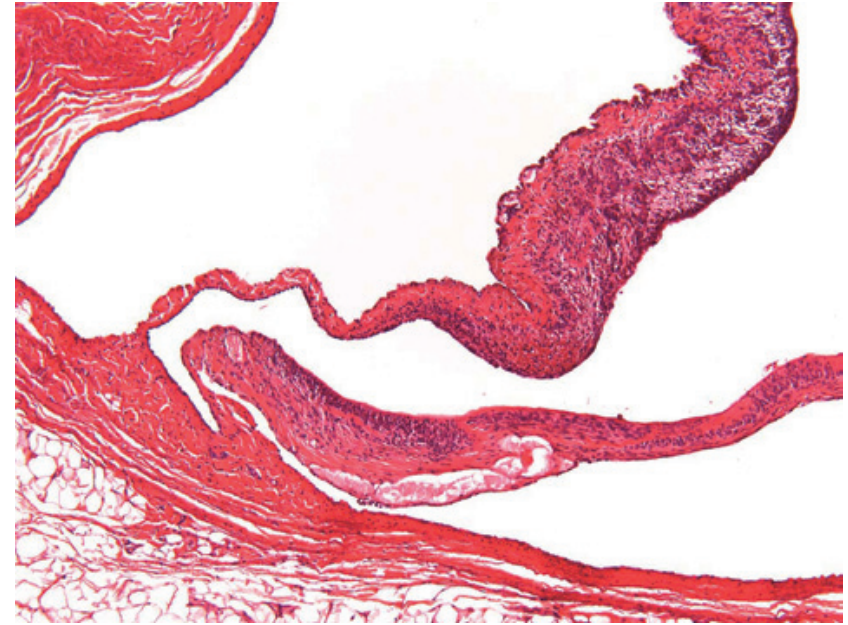

Fig. 19. Jugular vein near the tip of catheter with focally inflamed vein valve $(\operatorname{dog}, \mathrm{HE}$, lens $\times 10)$.

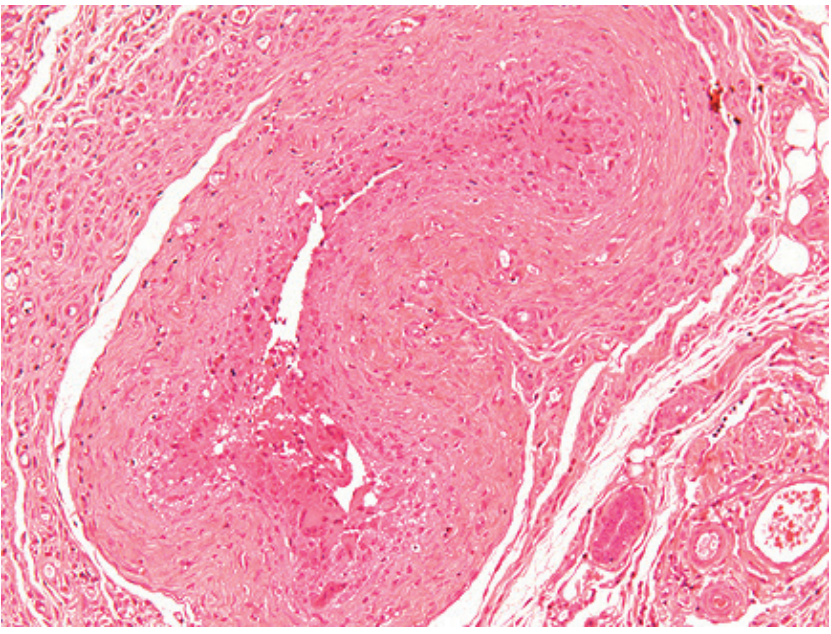

Fig. 20. Vena cava with minimal focal inflammation distal to tip of catheter (rat, HE, lens $\times 20$ ).

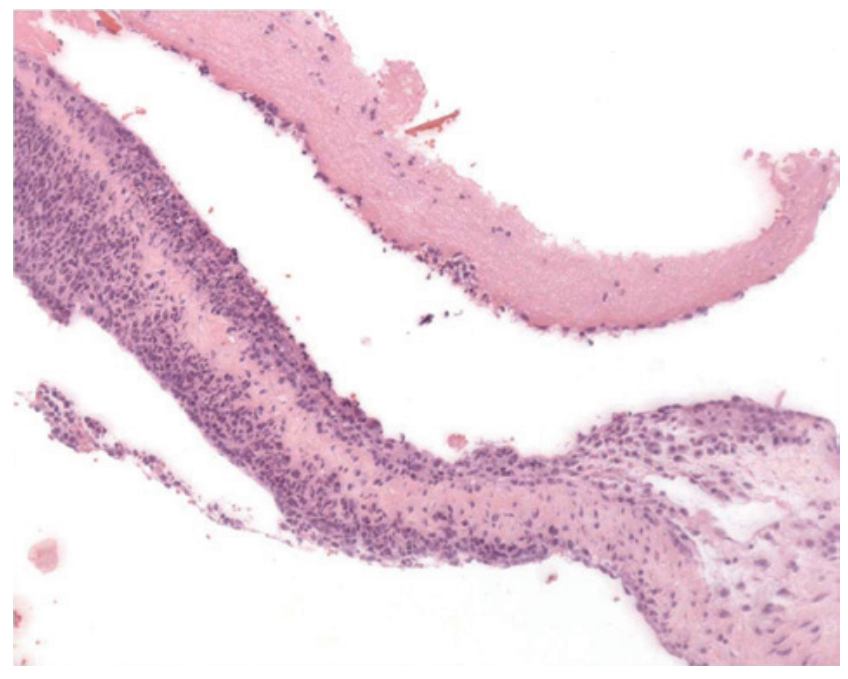

Fig. 21. Heart: valvular inflammation (rat, HE, lens $\times 20$ ). 


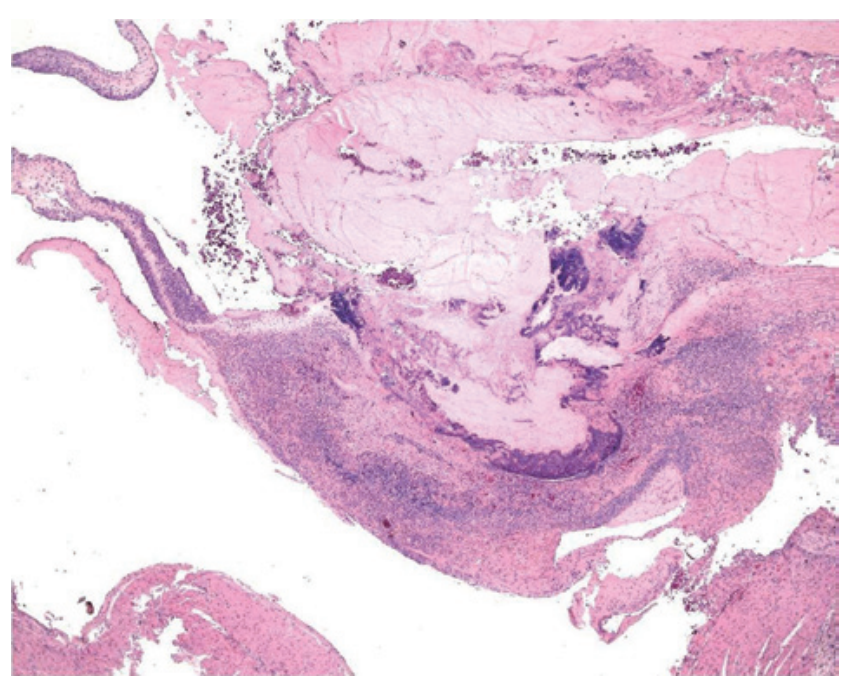

Fig. 22. Heart: valvular inflammation (rat, HE, lens $\times 10$ ).

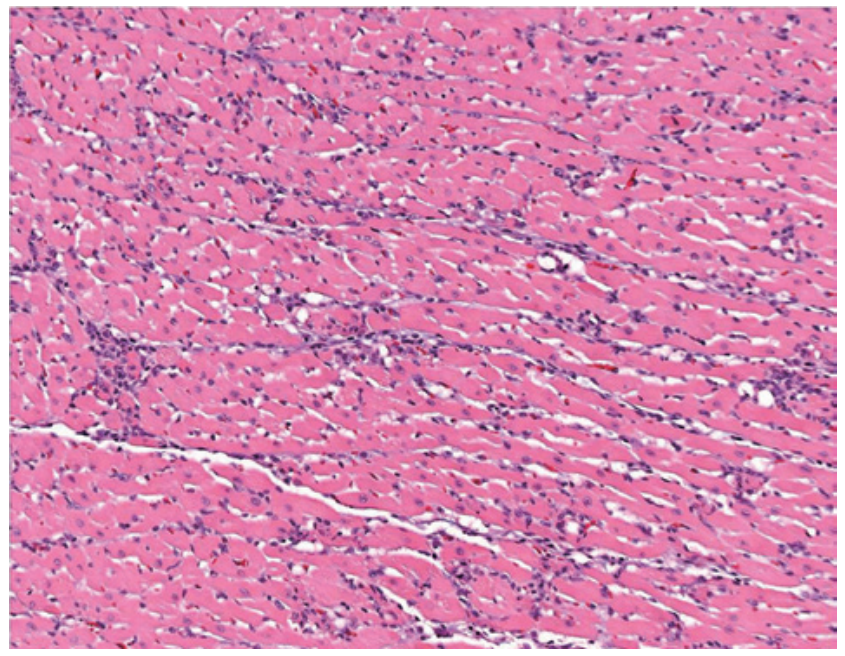

Fig. 23. Heart: myocardial degeneration characterized by mononuclear cell foci and multifocal fibrotic foci (rat, HE, lens $\times 20)$.

tissues or amorphous deposits attached to the vascular wall or within foci of granulomatous reactive inflammation.

The majority of lesions induced in continuous infusion studies however, are visible in other organs, and may represent deposition of the test item or a metabolite thereof (pigments, lipid droplets, crystalloids, crystal clefts etc.), inflammatory lesions in the terminal and smaller blood vessels and capillaries, increased embolism, changes in the heart including increased thrombus-related endo- (mainly valvular affection) and myocardial inflammation, effects on coronary arteries, increased incidence of endocardiosis (mainly related to increased thrombus formation or disseminated intravascular coagulopathy), dysthrombopoiesis and other unusual and rare lesions can also be seen.

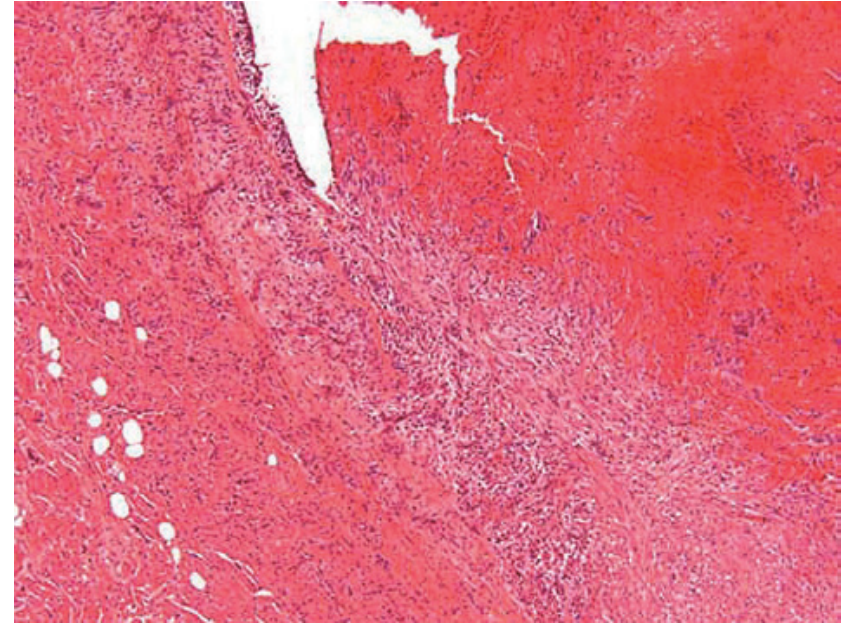

Fig. 24. Heart: organized thrombus with adjacent myocardial inflammation (dog, HE, lens $\times 10)$.

\section{Lesions in poorly performed studies}

Poor results in infusion studies are usually consequences of inadequate surgery, use of inappropriate materials or inadequate animal care during the in-life phase. All of these may cause excessive inflammatory changes including necrosis and suppuration along with excessive subacute to chronic active vasculitis, introduction of foreign bodies, up to a moderate abscess formation, tail necrosis in tail-cuff studies or tail cuffs placed too close to the rump causing severe necrosis in the gluteal region and finally, loss of catheters during the study. The interpretation of a study may become difficult or even impossible (Tables 1, 2). Besides non-aseptically and antiseptically performed surgical procedures including non-sterile 'from-roll' catheters, there may be other reasons e.g. traumatizing subcutaneous buttons, poor fixation of catheters by glue, misplaced catheters, poor fixation of catheters into blood vessels, subcutaneous looping of catheters and related ligature obstruction and destruction of blood vessels by sharp-end catheters

\section{Interpretation}

An important factor for the interpretation of results from large animal infusion studies is awareness of the test item's nature. The low number of individuals per sex and group in these studies may be problematic in respect of interpreting isolated findings in single animals.

In the first instance lumping of findings is more useful than splitting. For example, induced inflammatory changes in the lung would most likely not be recognized when splitting the term 'inflammation' into many other terms e.g. vascular inflammation, perivascular inflammation, focal extending inflammation, interstitial inflammation, thrombus-related inflammation etc. instead of using modifiers.

The pathologist may be a strong influencing variable depending on his or her experience, awareness of the modelrelated techniques and previous communication with study 
Table 1. Example for Abscess Formation at an Infusion Site Impossible to Interprete (Severity Graded in a Scale of Grades 1-5)

\begin{tabular}{|c|c|c|c|c|c|c|c|c|}
\hline \multirow{2}{*}{$\begin{array}{c}\text { Group } \\
\text { Number } \\
\text { Animals/Sex }\end{array}$} & \multicolumn{2}{|c|}{ Control } & \multicolumn{2}{|c|}{ Low dose } & \multicolumn{2}{|c|}{ Mid dose } & \multicolumn{2}{|c|}{ High dose } \\
\hline & $10 \mathrm{M}$ & $10 \mathrm{~F}$ & $10 \mathrm{M}$ & $10 \mathrm{~F}$ & $10 \mathrm{M}$ & $10 \mathrm{~F}$ & $10 \mathrm{M}$ & $10 \mathrm{~F}$ \\
\hline Grade 1 & - & & - & & - & & - & \\
\hline Grade 2 & 1 & & 2 & & 1 & & 1 & \\
\hline Grade 3 & 2 & & 1 & & - & & 1 & \\
\hline Grade 4 & - & & 4 & & 3 & & 2 & \\
\hline Grade 5 & 1 & & 7 & & 4 & & 2 & \\
\hline Total affected & 4 & & 4.3 & & 4.5 & & 6 & \\
\hline Mean grade & 3.3 & & 1.0 & & 1.6 & & 3.8 & \\
\hline
\end{tabular}

Table 2. Example for a Facilitated Interpretation of Induced Vasculitis at an Infusion Site (Severity Graded in a Scale of Grades 1-5)

\begin{tabular}{|c|c|c|c|c|c|c|c|c|}
\hline \multirow{2}{*}{$\begin{array}{c}\text { Group } \\
\text { Number }\end{array}$} & \multicolumn{2}{|c|}{ Control } & \multicolumn{2}{|c|}{ Low dose } & \multicolumn{2}{|c|}{ Mid dose } & \multicolumn{2}{|c|}{ High dose } \\
\hline & $10 \mathrm{M}$ & $10 \mathrm{~F}$ & $10 \mathrm{M}$ & $10 \mathrm{~F}$ & $10 \mathrm{M}$ & $10 \mathrm{~F}$ & $10 \mathrm{M}$ & $10 \mathrm{~F}$ \\
\hline Grade 1 & 2 & 3 & 2 & 3 & 2 & 1 & 1 & - \\
\hline Grade 2 & - & - & - & 3 & 3 & 2 & - & 2 \\
\hline Grade 3 & - & 1 & - & 1 & - & - & 4 & 2 \\
\hline Grade 4 & - & - & - & - & - & 2 & 2 & 2 \\
\hline Grade 5 & - & - & - & - & - & - & 4 & 3 \\
\hline Total affected & 3 & 4 & 2 & 7 & 5 & 5 & 11 & 9 \\
\hline Mean grade & 1.3 & 1.5 & 1.0 & 1.7 & 1.6 & 2.6 & 3.7 & 3.7 \\
\hline
\end{tabular}

directors, surgeons and technicians. The pathologist must be informed of any possible problems which occurred during the in-life part of the study as well as of any study-related information which may facilitate the interpretation of lesions recorded, i.e. body weight development, food consumption, clinical lesions and results of hematology, clinical biochemistry or even antibody formation etc. Furthermore, a good and complete documentation of the surgery including estimated blood loss, especially in rodents, is helpful at the end of the study and might deliver the key information to draw the right conclusions.

\section{Recommended Terminology}

The recommended nomenclature by Lilbert and Burnett ${ }^{5}$ for lesions recorded in infusion studies with monkeys, and by Morton et al. 7 for rats, were modified and complemented by the the ITO pathologists. The ITO recommends the use of the following terminology for all species used in continuous infusion studies:

\section{General:}

Inflammatory lesions are added by modifiers related to distribution (e.g. subcutaneous, intimal, adventitia etc.) and nature of the inflammatory process (e.g. acute, subacute, chronic active, purulent etc.). The grading is based on a scale of minimal, slight, moderate, marked, and severe (grades 1, 2, 3, 4, or 5, respectively), whereby minimal indicates the smallest visible effect on a defined section, severe the involvement of all visible tissue, moderate comprises approximately $50 \%$ of the visible tissue and the slight and marked are considered to be between these cases.

Port location or Perforation Site of Transcutaneous catheters:

- hemorrhage

- epidermal hyperplasia

- dermal or subcutaneous fibrosis

- capsule formation (port site)

- stitch granuloma

- dermal or subcutaneous inflammation

- pigment deposition, e.g. hemosiderin

Subcutaneous Tunnel:

- hemorrhage

- fibrosis

- capsule formation

- inflammation

- pigment deposition, e.g. hemosiderin

Vascular Location of Indwelling Catheter:

- hemorrhage

- fibrosis

- stitch granuloma

- vascular necrosis

- abscess

- vascular inflammation (alternatively phlebitis, arteritis)

- perivascular inflammation (alternatively periphlebi- 
tis, periarteritis)

- pigment deposition, e.g. hemosiderin

Vascular Tissues at Tip of Catheter and Distally to this Point:

- hemorrhage

- vascular fibrosis

- endothelial erosion

- endothelial proliferation

- medial thickening

- vascular necrosis

- abscess

- vascular inflammation (according to location, e.g., endophlebitis, phlebitis (panvasculitis))

- perivascular inflammation (alternatively periphlebitis, periarteritis)

- thrombus (free in vascular lumen)

- thrombovasculitis, e.g. thrombophlebitis (when thrombus is attached to vessel wall)

- pigment deposition, e.g. hemosiderin

\section{Discussion}

Little is published about common background lesions in different animal models used in continuous infusion studies $2,5-7$. Continuous infusion studies bear many possible problems affecting the study outcome. This depends on the species chosen, the choice of technical equipment, selection and performance of the surgical method, catheter material and calibre, preservation of catheter patency, as well as study related evaluation and interpretation.

In rats, a loss of animals of more than $5 \%$, including problems with the catheter, is not acceptable. Especially in this model, the background pathology should be within an acceptable range regarding inflammatory lesions. Excessively high incidences or severities of abscessation and/or necrosis cannot be considered to be normal and should lead to a very careful interpretation of such studies. Also, excessive traumatic dermal/tail lesions in studies performed with tail cuffs are often related to incorrect placement of the tail cuff. Expected lesions in tail cuff studies at the site of tail cuff fixation were published ${ }^{3}$ and consist of chronic inflammation extending into the tail muscles, as shown by the presence of fibrous tissue associated with muscle degeneration.

Another problem that might be encountered, mainly in small animal models when using the femoral vein as the primary site of infusion, is placement of the catheter too far distally into the vena cava, which may cause problems in study interpretation due to method related vascular lesions.

Although beagle dogs, pigs and monkeys are the largest laboratory animals used in this study type, and may be considered as a suitable research model, their behavior may affect the study outcome. Dogs and monkeys are poor patients, and their playing, biting and scratching occasionally cause trauma-related lesions at the site of the subcutaneously implanted port system, the subcutaneous catheter tunnel, the point where the catheter enters the blood vessel or the catheter tip. They may also pull out the catheter when possible.

The ranges of lesions observed in different species is similar between different laboratory species ${ }^{2,5,6}$. The incidence of thromboemboli in the lung of the rats is usually slightly below those in primates and the highest incidence is reported for $\operatorname{dog} s^{2,6}$, although the presence of venous thrombi at the catheter entry point is similar for all species. It was assumed, that the larger diameter of the jugular vein in the dog with its greater turbulence of blood flow possibly predisposes to fragmentation and dispersal of thrombi ${ }^{6}$.

Volume or flow rate ${ }^{7}$ have no major influence on the incidence of vascular and related lesions, whereas the catheter material may have an influence (sharp ends, material that getting brittle over time etc.). Severe lesions such as abscesses are rarely reported in control animals ${ }^{2}$.

When using the jugular vein as the primary vessel, the introduction of catheters too far proximally may cause irritation of the cardiac endothelium, enhancing secondary inflammatory lesion by thrombosis. In rat studies performed at Harlan Laboratory Ltd., Switzerland, the femoral vein did not reveal such lesions at a similar incidence.

The catheter material should be checked prior to testing for compatibility with the test item.

At the end of the scheduled study periods, careful necropsy may be helpful in differentiating mechanically induced, traumatic or post-surgical conditions from normal background and treatment-related lesions. These observations may, in some circumstances, be supported by in-life observations, i.e. recording of clinical symptoms (e.g. lameness), or X-rays along with contrast media (e.g. vascular necrosis).

High activity of the endothelium attempting to cover the catheter's luminal surfaces may cause focal problems leading to blockage of the catheter, although this is rare. Even rarer is an effect of thrombosis on the catheter's patency.

Infectious lesions are rarely encountered under normal laboratory conditions while following the principles of antiseptic and aseptic surgical conditions.

It is highly recommended to sample tissues at the following locations in small and large animals:

- dermal subcutaneous tissue around the implanted port or the site of catheter perforation of the skin in studies where no port access system was used,

- at least one sample of tissue surrounding the subcutaneous tunnel at the course of catheter,

- vascular and surrounding tissues at the point where the catheter enters into the blood vessel,

- vascular and surrounding tissues at the tip of catheter, and

- two samples for:

rodents and rabbits at a distance of approximately: 0.3 and 0.5 to $1.0 \mathrm{~cm}$ distally to the point of delivery. non-rodents: 0.5 and $1.0 \mathrm{~cm}$ distally to the point of 
delivery

- control vessel (Figs. 3, 4).

The lesions that may be recorded at each of these sites are very consistent and vary within a small range under well-established study conditions. Post-surgical lesions include fibrosis around the implanted port and in tissues surrounding the subcutaneous tunnel, inflammatory changes at minor degrees at the latter site as well the indwelling point of the catheter into the blood vessel, minor endovascular fibrosis along the course of the catheter, minimal vascular inflammation around the tip of catheter including peri-/vasculitis, endothelial proliferation, thrombosis, focal vein valve inflammation etc. Distal to the point of product delivery (tip of catheter), such lesions should be less in severity or not present at all.

\section{Conclusion}

In accordance with a proposed nomenclature for vascular lesions established in primate studies 5 , ITO proposes a standard terminology for lesions at all sites in contact with the delivery system (port, catheter) and a protocol for samples to be collected during necropsy. This recommendation includes all used animal models.

\section{References}

1. Fredenburg, N. Evaluation of Catheter Tip Configuration and Lock Solutions in Rat Cannulation Models, Harlan 2008 (internal investigation).

2. Dawson S, Alys E, and Bradley AE. Long-term intravenous catheters. ESTP CDRom Classical Examples of Toxicologic Pathology. 3rd ed.

3. de Jong WH, Timmerman A, and van Raaij MTM. Longterm cannulation of the vena cava of rats for blood sampling: local and systemic effects observed by histopathology after six weeks of cannulation. Lab Anim. 35: 243-248. 2001.[Medline] [CrossRef]

4. Harlan Laboratories Ltd. Blumbach K, Kaiser S. Validation Study. Internal Report. 2010.

5. Lilbert J, and Burnett R. Main vascular changes seen in the saline controls of continuous infusion studies in the cynomolgus monkey over an eight-year period. Toxicol Pathol. 31: 273-280. 2003.[Medline]

6. Lilbert J, and Mowat V. Common vascular changes in the jugular vein of saline controls in continuous infusion studies in the Beagle dog. Toxicol Pathol. 32: 694-700. 2004. [Medline] [CrossRef]

7. Morton D, Safron JA, Glosson J, Rice DW, Wilson DM, and White RD. Histologic lesions associated with intravenous infusions of large volumes of isotonic saline solution in rats for 30 days. Toxicol Pathol. 25: 390-394. 1997.[Medline] [CrossRef]

8. VanWijk H. Personal communication, 2010.

9. Weber K. Suture materials. Internal Report. Harlan, 2006. 